Doi: $10.15863 /$ TAS

\section{International Scientific Journal}

\section{Theoretical \& Applied Science}

p-ISSN: 2308-4944 (print) e-ISSN: 2409-0085 (online)

Year: 2014 Issue: 12 Volume: 20

Published: 30.12 .2014 http://www.T-Science.org
Vadim Nikolaevich Lesev

Candidate of Physical and Mathematical Sciences, Chief of the Department of Differential Equations of Kabardino-Balkarian State University, Russia diff@,kbsu.ru

Maryana Adibovna Shardanova Undergraduate of mathematical faculty of Kabardino-Balkarian State University, Russia shardanova2010@yandex.ru

SECTION 1. Theoretical research in mathematics.

\title{
ABOUT SOLVABILITY OF THE BOUNDARY VALUE PROBLEMS FOR THE INHOMOGENEOUS EQUATION OF HING ORDER WITH VARIABLE COEFFICIENTS
}

Abstract: The solvability of the classical edge task for the inhomogeneous equation in partial fourth-order derivatives has been proven. The method of the finite integral transformations has been used to prove the existence of the solution.

Key words: high-order equation, edge task, proof of the existence of solution method of finite integral transformations.

Language: Russian

Citation: Lesev VN, Shardanova MA (2014) ABOUT SOLVABILITY OF THE BOUNDARY VALUE PROBLEMS FOR THE INHOMOGENEOUS EQUATION OF HING ORDER WITH VARIABLE COEFFICIENTS. ISJ Theoretical \& Applied Science 12 (20): 101-103. http://dx.doi.org/10.15863/TAS.2014.12.20.22

\section{О РАЗРЕШИМОСТИ КРАЕВЫХ ЗАДАЧ ДЛЯ НЕОДНОРОДНОГО УРАВНЕНИЯ ВЫСОКОГО ПОРЯДКА С ПЕРЕМЕННЫМИ КОЭФФИЦИЕНТАМИ}

Аннотация: В работе доказана разрешимость классических краевых задач для неоднородного уравнения в частных производных четвертого порядка. Для доказательства существования решения использован метод конечных интегральных преобразований.

Ключевые слова: уравнения высокого порядка, краевая задача, доказательство существования решения, метод конечных интегральных преобразований.

\section{ВВЕДЕНИЕ}

На сегодняшний день, в математической литературе имеется достаточно работ в которых исследуются задачи [1-7] для уравнений четвертого порядка. Широкий анализ публикаций по данному направлению проведен в работе [8].

Настоящая работа посвящена исследованию вопроса разрешимости краевых задач для уравнения четвертого порядка с переменными коэффициентами в прямоугольной области посредствам конечных интегральных преобразований Фурье.

\section{ПОСТАНОВКА ЗАДАЧИ}

В области $\Omega=\{z: 0<x<\ell, 0<t<h\}$ евклидовой плоскости точек $z=(x, t)$ рассмотрим уравнение

$$
L u=F(x, t),
$$

где $\ell, h$ - положительные величины,

$$
\begin{aligned}
& L=\frac{\partial^{4}}{d x^{4}}+a(t) \cdot \frac{\partial^{4}}{d x^{2} d t^{2}}+b(t) \cdot \frac{\partial^{4}}{\partial t^{4}}+ \\
& +c(t) \cdot \frac{\partial^{3}}{\partial t^{3}}+d(t) \cdot \frac{\partial^{3}}{\partial x^{2} d t}+e(t) \cdot \frac{\partial^{2}}{\partial x^{2}}+ \\
& +f(x) \cdot \frac{\partial^{2}}{\partial t^{2}}+g(t) \cdot \frac{\partial}{\partial t}+h(t),
\end{aligned}
$$

$a(t), b(t), \ldots F(x, t)$ - заданные непрерывные функции.

Обозначим через $J$ отрезок $(0,1)$ оси абсцисс.

Задача А1. Найти регулярное в области $\Omega$ решение $u(x, t)$ уравнения (1) из класса 
$C^{2}(\bar{\Omega}) \cap C_{t}^{4}(\Omega \cup \bar{J}), \quad$ удовлетворяющее

краевым условиям

$$
\begin{aligned}
& u(0, t)=\varphi_{1}(t), \quad u(\ell, t)=\psi_{1}(t), \\
& u_{x x}(0, t)=\varphi_{2}(t), \quad u_{x x}(\ell, t)=\psi_{2}(t), \\
& u(x, 0)=\gamma_{1}(x), \quad u_{t}(x, 0)=\gamma_{2}(x), \\
& u_{t t}(x, 0)=\gamma_{3}(x), \quad u_{t t t}(x, 0)=\gamma_{4}(x),
\end{aligned}
$$

и условиям согласования $\varphi_{1}(0)=\gamma_{1}(0)$, $\gamma_{1}(l)=\psi_{1}(0)$, где $\varphi_{i}(t), \psi_{i}(t),(i=1,2)$ и $\gamma_{j}(t),(j=\overline{1,4})$ - заданные достаточно гладкие функции.

Задача А2. Найти регулярное в области $\Omega$ решение $u(x, t)$ уравнения (1) из класса $C^{3}(\bar{\Omega}) \cap C_{t}^{4}(\Omega \cup \bar{J})$, удовлетворяющее всем условиям задачи $\mathrm{A}_{1}$, кроме условий (2), (3), которые заменены условиями

$$
\begin{array}{cc}
u_{x}(0, t)=\varphi_{1}(t), & u_{x}(\ell, t)=\psi_{1}(t), \\
u_{x x x}(0, t)=\varphi_{2}(t), & u_{x x x}(\ell, t)=\psi_{2}(t) .
\end{array}
$$

\section{ДОКАЗАТЕЛЬСТВО РАЗРЕШИМОСТИ \\ ЗАДАЧИ}

Остановимся на исследовании задачи $\mathrm{A}_{1}$ более подробно. Применяя к уравнению (1) конечное синус-преобразование Фурье по переменной $x$ [9.стр.75]:

$$
S[u]=\frac{2}{\ell} \int_{0}^{\ell} u(x, t) \sin \left(\frac{n \pi x}{\ell}\right) d x
$$

и принимая во внимание условия (2), (3), получим

$$
\begin{aligned}
& b(t) \cdot \frac{d^{4} \bar{u}_{n}}{d t^{4}}+c(t) \cdot \frac{d^{3} \bar{u}_{n}}{d t^{3}}+p(t) \cdot \frac{d^{2} \bar{u}_{n}}{d t^{2}}+ \\
& +q(t) \cdot \frac{d \bar{u}}{d t}+r(t) \cdot \bar{u}_{n}=s(t),
\end{aligned}
$$

где

$$
\begin{aligned}
& p(t)=f(t)-a(t) \cdot\left(\frac{n \pi}{l}\right)^{2}, \\
& q(t)=g(t)-d(t) \cdot\left(\frac{n \pi}{l}\right)^{2},
\end{aligned}
$$

$$
\begin{aligned}
& r(t)=h(t)+\left(\frac{n \pi}{l}\right)^{4}-e(t) \cdot\left(\frac{n \pi}{l}\right)^{4}, \\
& s(t)=\bar{F}(t)+\frac{2 n \pi}{l^{2}} \cdot\left\{\left[\varphi_{1}(t)+(-1)^{n+1} \cdot \psi_{1}(t)\right] .\right. \\
& \cdot\left[\frac{(\pi n)^{2}}{l}-e(t)\right]-\varphi_{2}(t)+(-1)^{n} \cdot \psi_{2}(t)-a(t) \cdot \\
& \cdot \frac{d^{2}}{d t^{2}} \cdot\left[\varphi_{1}(t)+(-1)^{n+1} \cdot \psi_{1}(t)\right]-d(t) \cdot \frac{d}{d t} . \\
& \left.\cdot\left[\varphi_{1}(t)+(-1)^{n+1} \cdot \psi_{1}(t)\right]\right\},
\end{aligned}
$$

$\bar{u}_{n}(t), \bar{F}(t)$-результат преобразования функций $u(x, t)$ и $F(x, t)$ соответственно. Коэффициенты и правые части уравнений (6) непрерывны, а следовательно по хорошо известной теореме об общем решении неоднородного дифференциального уравнения, например [10. стр. 107], соответствующие им общие решения могут быть представлены в виде:

$$
\bar{u}_{n}(t)=\Phi\left(t, n, \lambda_{1}, \lambda_{2}, \lambda_{3}, \lambda_{4}\right),
$$

где $\lambda_{i}(i=\overline{1,4})-$ произвольные постоянные нуждающиеся в определении.

Замечание 1. Представление (7) имеет место при $b(t) \neq 0$ и $c(t) \neq 0$. Случаи, когда:

1) $b(t)=0, c(t) \neq 0$;

2) $b(t)=c(t)=0$

требуют особого рассмотрения, т.к. тогда уравнение (6) вырождается в уравнение третьего или второго порядка соответственно, a следовательно задачи $\mathrm{A}_{1}, \mathrm{~A}_{2}$ становятся переопределенными. Поэтому в первом случае для устранения некорректности задач надо вывести из рассмотрения одно, а во втором - два из условий входящих в (4).

Для получения соотношений позволяющих определить постоянные $\lambda_{i}(i=\overline{1,4})$ применим (5) к граничным условиям (4), будем иметь: 


$$
\begin{aligned}
& \bar{u}_{n}(0)=\frac{2}{l} \int_{0}^{l} \gamma_{1}(x) \sin \left(\frac{n \pi x}{l}\right) d x, \\
& \left.\frac{d \bar{u}_{n}}{d t}\right|_{t=0}=\frac{2}{l} \int_{0}^{l} \gamma_{2}(x) \sin \left(\frac{n \pi x}{l}\right) d x, \\
& \left.\frac{d^{2} \bar{u}_{n}}{d t^{2}}\right|_{t=0}=\frac{2}{l} \int_{0}^{l} \gamma_{3}(x) \sin \left(\frac{n \pi x}{l}\right) d x, \\
& \left.\frac{d^{3} \bar{u}_{n}}{d t^{3}}\right|_{t=0}=\frac{2}{l} \int_{0}^{l} \gamma_{4}(x) \sin \left(\frac{n \pi x}{l}\right) d x,
\end{aligned}
$$

Удовлетворяя (7) условиям (8), находим соотношения для постоянных $\lambda_{i}(i=\overline{1,4})$. Таким образом, вопрос разрешимости задачи $\mathrm{A}_{1}$ эквивалентно редуцирован к вопросу разрешимости последовательности ОДУ для коэффициентов Фурье. Применяя обратное конечное синус-преобразование, получаем решение задачи $\mathrm{A}_{1}$ в области $\Omega$ в виде:

$$
u(x, t)=\sum_{n=1}^{\infty} \bar{u}_{n}(t) \cdot \sin \left(\frac{n \pi x}{\ell}\right) .
$$

Замечание 2. Задача $\mathrm{A}_{2}$ исследуется аналогично, но вместо преобразования (5) используется конечное косинус-преобразование Фурье.

Замечание 3. Начальные условия (4) могут быть заменены любыми другими, позволяющими однозначно находить частные решения соответствующих задач для уравнения (6).

\section{References:}

1. Lesev VN, Shardanova MA (2014) Primenenie metoda konechnykh integral'nykh preobrazovaniy $\mathrm{k}$ issledovaniyu kraevoy zadachi dlya uravneniya vysokogo poryadka. ISJ Theoretical \& Applied Science. 2014. № 5 (13). - pp. 1-4. doi: http://dx.doi.org/10.15863/TAS.2014.05.13.1

2. Lesev VN, Shardanova MA (2014) Issledovanie razreshimosti klassicheskoy kraevoy zadachi dlya neodnorodnogo uravneniya chetvertogo poryadka // Materialy IV mezhdunarodnoy nauchno-prakticheskoy konferentsii «Aktual'nye napravleniya fundamental'nykh prikladnykh issledovaniy. 2014. Tom 1. - pp. 200-201.

3. Dumaeva LV, Lesev VN (2006) Lokal'naya kraevaya zadacha dlya neodnorodnogo uravneniya giperbolicheskogo tipa chetvertogo poryadka // Tezisy dokladov Vserossiyskoy nauchnoy konferentsii studentov, aspirantov, i molodykh uchenykh. 2006. T.2. - pp. 239-242.

4. Ayshaev KM, Lesev VN (2007) K teorii nelineynykh uravneniy vysokogo poryadka// Materialy Mezhdunarodnogo kongressa studentov, aspirantov i molodykh uchenykh: Perspektiva - 2007. Nal'chik. - pp.162-163.

5. Amanov D, Murzambetova MB (2013) Kraevaya zadacha dlya uravneniya chetvertogo poryadka s mladshim chlenom // Vestnik udmurtskogo universiteta. Matematika. Mekhanika. Komp'yuternye nauki, 2013. Vyp. 1. - pp. 3-10.

6. Eleev VA, Laypanova AM, Lesev VN (2008) O razreshimosti kraevoy zadachi dlya smeshannogo uravneniya metodom konechnykh integral'nykh preobrazovaniy v pryamougol'noy oblasti // Vestnik Kabardino-Balkarskogo gosudarstvennogo universiteta. Seriya matematicheskie nauki. 2008. V. 5. - pp. 32-35.

7. Lesev VN (2006) Issledovanie razreshimosti kraevykh zadach dlya uravneniya chetvertogo poryadka metodom konechnykh integral'nykh preobrazovaniy // Materialy mezhdunarodnoy konferentsii: Sovremennye problemy matematiki. 2006. - pp.44-46.

8. Dzhuraev TD, Sopuev A (2000) K teorii differentsial'nykh uravneniy $\mathrm{V}$ chastnykh proizvodnykh chetvertogo poryadka. Tashkent: Fan, - 144.

9. Farlou S (1985) Uravneniya s chastnymi proizvodnymi dlya nauchnykh rabotnikov i inzhenerov: Per. s angl. - Moscow: Mir. 1985. -384 .

10. El'sgol'ts LE (1969) Differentsial'nye uravneniya i variatsionnoe ischislenie. Moscow: Nauka.- 424 